\title{
Case Study of ETL Java Code Generation from Domain Specific Language
}

\author{
Sunisa Junsawang and Yachai Limpiyakorn ${ }^{+}$ \\ Department of Computer Engineering, Chulalongkorn University, Bangkok 10330, Thailand
}

\begin{abstract}
Extract-Transform-Load, ETL, is the integration layer in data warehouse environment. The quality of ETL contributes to the accuracy and the correctness of data, it is expensive, though. This paper aims to improve the process productivity and usability. A domain-specific language has been developed for scripting ETL processes. Alternatively, the input of ETL scripts written by non-technical users in Excel format is also allowed. The paradigm of code-to-code transformation is applied for transforming the textual, domain-specific language into Java code. The etIDSL code generator is implemented with MVC architecture design. A case study and the user evaluation of the proposed approach are also discussed. The overall rating is satisfaction.
\end{abstract}

Keywords: domain specific language, ETL, query, data warehouse, software process improvement.

\section{Introduction}

In data warehouse environment, Extract-Transform-Load (ETL) processes constitute the integration layer responsible for pulling data from data sources to targets, via a set of transformations. ETL is a critical component contributes to the accuracy and the correctness of data, which are key factors of the success or failure of data warehouse projects. However, building ETL processes consumes up to $70 \%$ of resources regarding time, money and effort [1]. To improve the process productivity and usability, we develop a domain-specific language (DSL) for scripting the ETL process. The prototyping code generator, etlDSL, is implemented to support separation of concerns by means of Model-View-Controller (MVC) architecture design as described in the earlier work [2]. In this paper, the Interface layer of the View component is enhanced with the provision of Excel templates for user-defined ETL scripts to promote the usability. A case study and the user evaluation of the proposed approach are also discussed.

In literature, Hemel et al. [3] presented a case study of the code generation by model transformation approach applied in the development of WebDSL, which is a domain-specific language for dynamic web applications. The WebDSL generator follows the four-level model organization to transform high-level models into Java code and XML files. Oliveira and Belo [4] proposed the use of Business Process Modelling Notation (BPMN) for building the simulator of ETL process. The DSL grammars were defined and translated to Java language by Xtext tool. Deneke [5] developed the DSL by C\# for building ETL workflows. The Object-Relational Mapper (ORM) was used for data gathering instead of data query with SQL. Lasya and Tanuku [6] proposed an approach to optimizing the executable code generated from a certain SQL query. Several alternative query trees are constructed based on the relational algebra expressions underlying the input SQL query. The Query Execution Plan associated with the query tree that provides the minimum cost will be selected for executable code generation. Santos and Belo [7] proposed modelling ETL conciliation tasks using relational algebra operators. Several scenarios of ETL were illustrated in relational algebra trees for integrity awareness.

+ Corresponding author. Tel.: + 6682218 6959; fax: +66822186955.

E-mail address:Yachai.L@chula.ac.th. 


\section{2. etIDSL}

The DSL commands contain the common vocabularies familiar to the domain experts. In earlier work [2], we described the development of a domain-specific language created for scripting ETL processes. The current DSL presented in this paper has been slightly improved. Fig. 1 illustrates its meta-model which is a grammar describing the valid sentences of the language. The etIDSL code generator is also implemented providing alternative input of ETL scripts written by non-technical users, such as Business Analyst (BA). The overview of the proposed ETL process is depicted in Fig. 2.

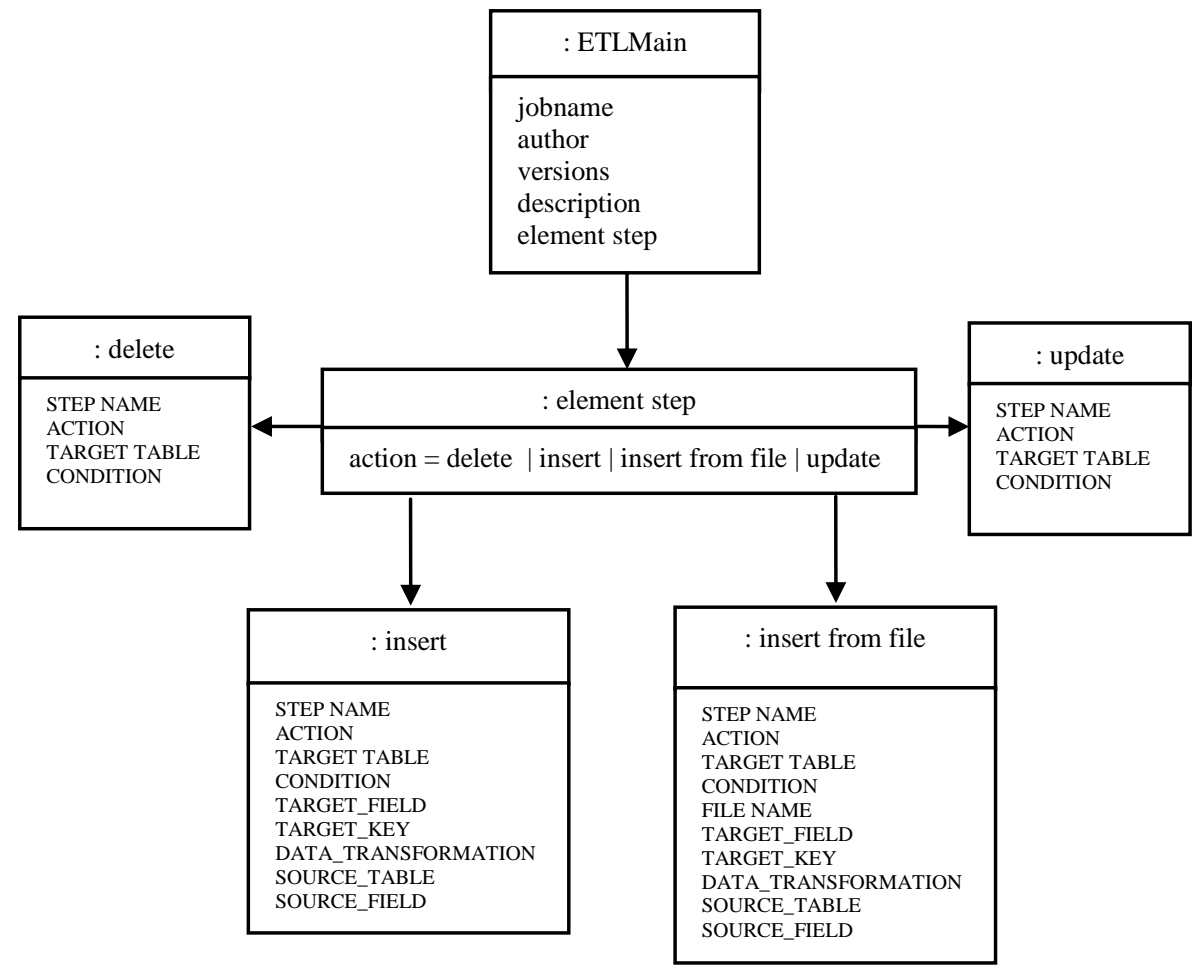

Fig. 1: DSL Meta-model.

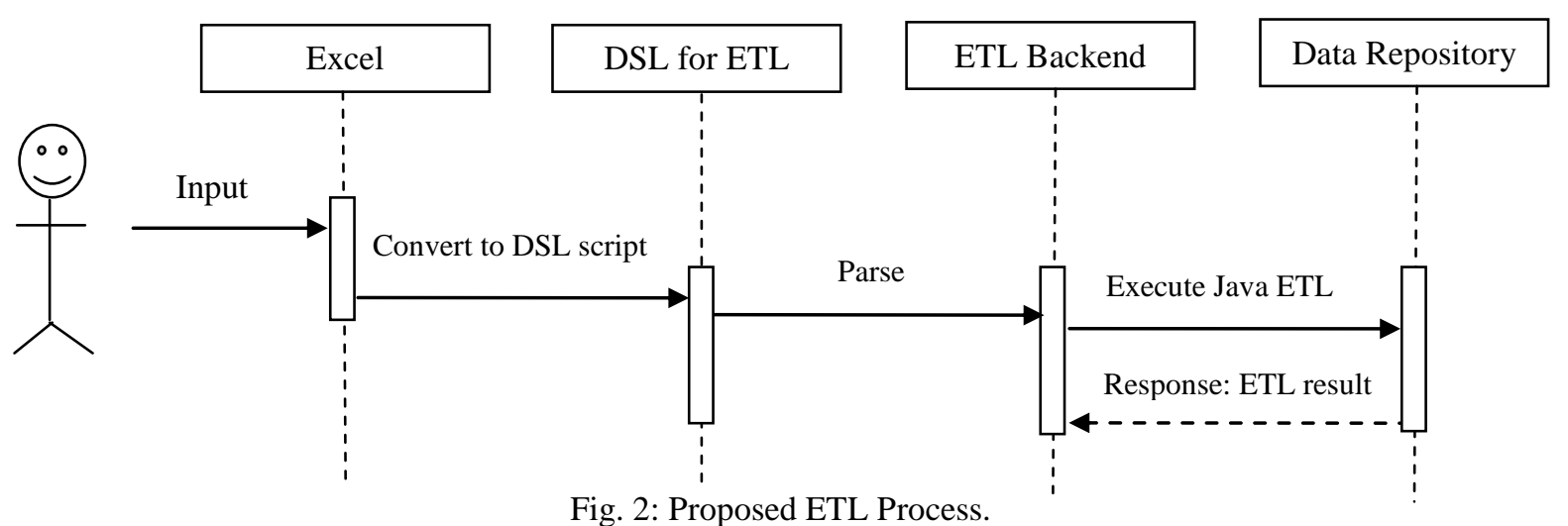

Fig. 2: Proposed ETL Process.

Non-technical users can traditionally describe ETL processes in Excel files and then save to .etl extension, i.e. automatically convert to DSL script. Alternatively, they can directly write the DSL scripts. As shown in Fig. 3, the example ETL script contains Action delete and insert into the target table CAMPAIGN_FACT with the updates. The data transformation is categorized into four types:

1) Constraint: e.g. move direct, null.

2) Function: e.g. nvl(), sum().

3) Number: e.g. $0,1$.

4) String: e.g. 'USE'. 


\begin{tabular}{|c|c|c|c|c|}
\hline STEP NAME & \multicolumn{4}{|c|}{ Delete CAMPAIGN } \\
\hline ACTION & \multicolumn{4}{|c|}{ delete } \\
\hline TARGET TABLE & \multicolumn{4}{|c|}{ CAMPAIGN_FACT } \\
\hline CONDITION & \multicolumn{4}{|c|}{$\begin{array}{l}\text { (STORE,DT_KEY) in } \\
\text { (select } 1 . S T O R E, 1 . D T \_K E Y \\
\text { from campaign_fct c, STG_LOG } 1 \\
\text { where c.STORE = 1.STORE } \\
\text { and c.DT_KEY = 1.DT_KEY) }\end{array}$} \\
\hline STEP NAME & \multicolumn{4}{|c|}{ CAMPAIGN use_cards } \\
\hline ACTION & \multicolumn{4}{|l|}{ insert } \\
\hline TARGET TABLE & \multicolumn{4}{|c|}{ CAMPAIGN_FACT (update and insert) } \\
\hline CONDITION & \multicolumn{4}{|c|}{$\begin{array}{l}\text { sale_attribute.saleheaderid }=\text { sales_header_fct.sale_id } \\
\text { and sales_header_fct.store }=\text { store_dim.store } \\
\text { and sale_attribute.att } 056=\text { '2' } \\
\text { group by sale_attribute.saleheaderid, } \\
\text { to_char(to_date(substr(sale_attribute.att } 040,1,10) \text {,'yyyy-mm- } \\
\text { dd'),'yyyymmdd'), } \\
\text { sales_header_fct.dt_key, } \\
\text { sales_header_fct.store, } \\
\text { store_dim.store_nm, } \\
\text { sales_header_fct.channel_sr_key, } \\
\text { sale_attribute.att039,sale_attribute.ATT054 ,sale_attribute.ATT055, } \\
\text { sale_attribute.ATT057) }\end{array}$} \\
\hline TARGET_FIELD & TARGET_KEY & DATA_TRANSFORMATION & SOURCE_TABLE & SOURCE_FIELD \\
\hline DT_KEY & & move direct & sales_header_fct & dt_key \\
\hline CHANNEL_SR_KEY & & move direct & sales_header_fct & channel_sr_key \\
\hline STORE & key & move direct & sales_header_fct & store \\
\hline STORE_NM & & move direct & store_dim & store_nm \\
\hline CARD_NUMBER & key & move direct & sale_attribute & att046 \\
\hline SALE_CARD_SALEID & key & move direct & sale_attribute & saleheaderid \\
\hline USE_CARD_SALEID & & null & & \\
\hline SALE_CARD_QTY & & 0 & & \\
\hline USE_CARD_QTY & & nvl(sum(att043),0) & & \\
\hline TRUN_TYPE & & 'USE' & & \\
\hline DATA_LOAD_DT & & sysdate & & \\
\hline DATA_UPDT_DT & & sysdate & & \\
\hline ATTR & & move direct & sale_attribute & ATT054 \\
\hline ATTR2 & & move direct & sale_attribute & ATT055 \\
\hline ATTR3 & & move direct & sale_attribute & ATT057 \\
\hline ATTR4 & & ' ' & & \\
\hline ATTR5 & & ' ' & & \\
\hline NUM_SALE_CARD_QTY & & 0 & & \\
\hline NUM_USE_CARD_QTY & & 1 & & \\
\hline
\end{tabular}

Fig. 3: Example ETL Script in Excel File.

Next, Xtext, an open-source framework for developing programming languages and domain-specific languages, is used as the parser generator. The DSL script will be parsed to Java code. Fig. 4 illustrates the DSL script associated with the ETL Excel script shown in Fig. 3. The execution of Java code will then invoke the ETL backend to create SQL statements using a set of predefined transformation rules, examples as shown in Fig. 5. The generation of SQL statements in this work supports full outer join, inner join, left join, and right join. Finally, the target model will be created by merging all the created target meta-models with the predefined templates of standard Java commands and the log reporting the execution status. 


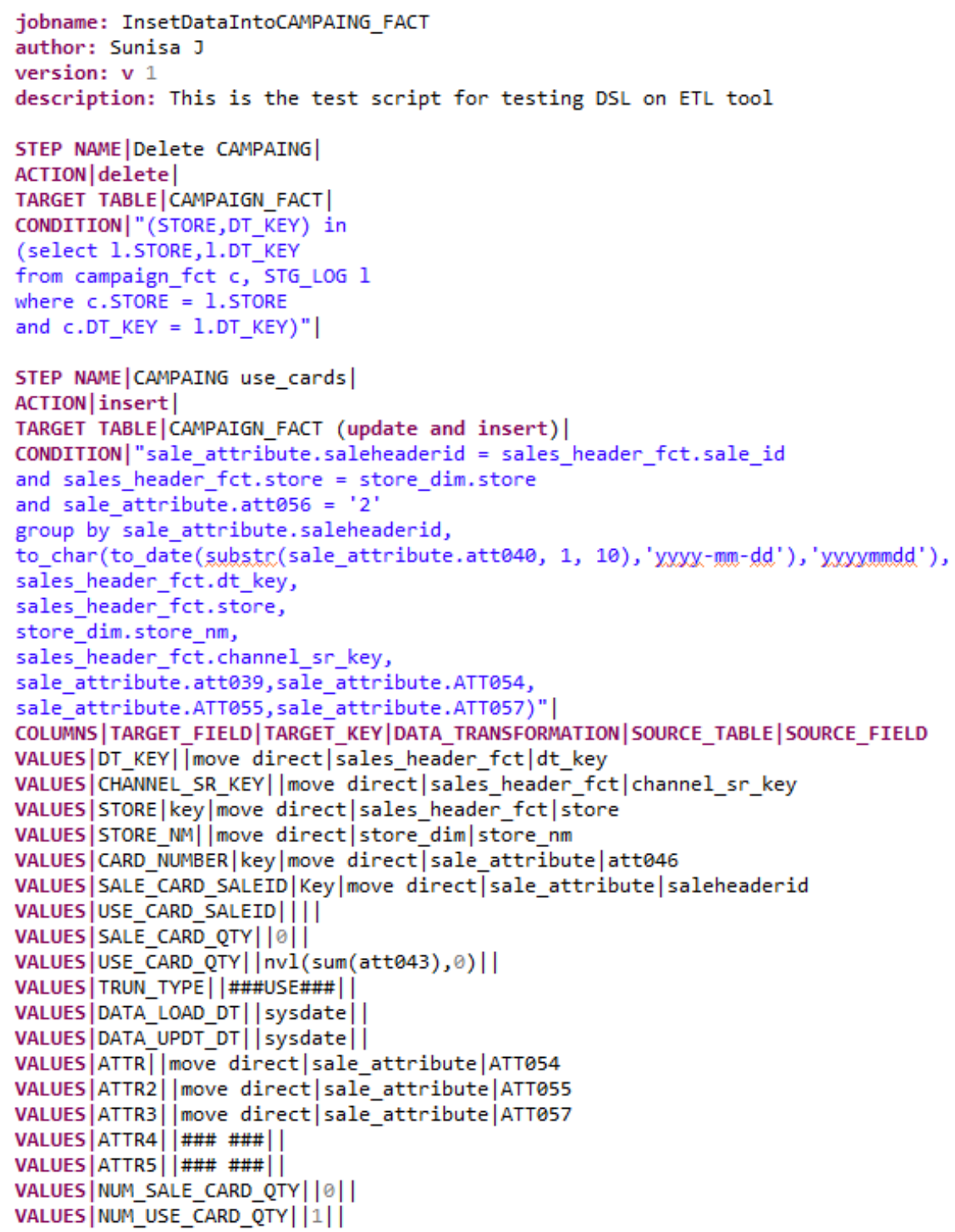

Fig. 4: DSL Script Associated with ETL Excel File in Fig. 3.

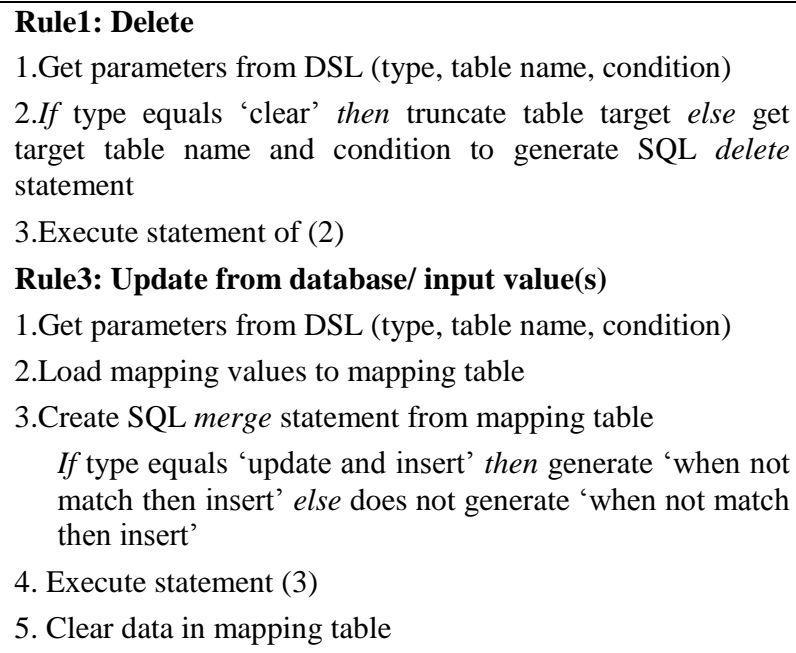

\section{Rule2: Insert from file}

1. Get parameters from DSL (type, table name, file name, mapping values)

2. Load mapping values to mapping table

3. Create temporary table based on column of file

4. Load file to temporary table

5.Generate SQL statement from data in mapping table

6. If type equals 'clear and insert' then delete data in target table and generate SQL insert statement on data in mapping table else generate SQL insert statement on data in mapping table

7.Execute statement of (6)

8.Drop temporary table and clear data in mapping table

Fig. 5: Example SQL Transformation Rules.

\section{Evaluation}

The satisfaction survey was conducted based on the quality dimensions including: Operability (users' efforts for operation and operation control), Learnability (how well users can learn the application of 
software), Correctness (if the ETL script functions correctly), Fitness for Use (if the system fulfills intended use), and Overall Satisfaction. Six people who are project manager, business analysts, tester, programmer, and QA were selected for writing the DSL script (Fig. 4). Table 1 summarizes the results reporting the overall averaging score equals to 4.07 or achieving satisfaction ranking.

Table 1: Satisfaction Survey Results.

\begin{tabular}{|l|c|c|c|c|c|c|}
\hline & \multicolumn{7}{c|}{ Score } & \multirow{2}{*}{ Average Score } \\
\cline { 2 - 8 } & $\mathbf{5}$ & $\mathbf{4}$ & $\mathbf{3}$ & $\mathbf{2}$ & $\mathbf{1}$ & 0 \\
\hline Operability & 4 & 1 & 1 & 0 & 0 & 4.5 \\
\hline Learnability & 1 & 4 & 1 & 0 & 0 & 5 \\
\hline Correctness & 6 & 0 & 0 & 0 & 0 & 3.17 \\
\hline Fitness for Use & 0 & 1 & 5 & 0 & 0 & 3.67 \\
\hline Overall Satisfaction & 0 & 4 & 2 & 0 & & $\mathbf{4 . 0 7}$ \\
\hline \multicolumn{7}{|c|}{} \\
\hline
\end{tabular}

Notes: Ranks: 1-very unsatisfaction; 2-unsatisfaction; 3-neutral; 4-satisfaction; 5-very satisfaction.

\section{Conclusion}

To support ETL layer in data warehouse environment, this paper presents the development of etlDSL generator to transform high-level abstractions into Java code. With Xtext framework, the proposed approach can be also applied for the transformation to other languages, such as C\#, .NET. This will require the modification of ETL Backend to support word truncation in other programming languages. A case study of ETL written in DSL is discussed and evaluated. The results showed that the users are satisfied with the proposed approach. The etIDSL system could function the ETL process correctly. The users can learn and operate the application software with not much effort. However, some user feedbacks suggest more pilot studies on existing ETL processes should be conducted before widely used.

\section{References}

[1] A. Kabiri and D. Chiadmi, "SURVEY ON ETL PROCESSES," Journal of Theoretical and Applied Information Technology, vol. 54, 2013.D. Ghosh, DSLs in Action: Manning Publications, 2011.

[2] S. Junsawang and Y. Limpiyakorn, "A Domain Specific Language for Scripting ETL Process," 2017.

[3] Z. Hemel, L. C. L. Kats, and E. Visser, "Code Generation by Model Transformation: A Case Study in Transformation Modularity," 2008.

[4] B. Oliveira and O. Belo, "On the specification of extract, transform, and load patterns behavior: A domain specific language approach," 2016.

[5] W. Deneke, "A Domain Specific Model for Generating ETL Workflows from Business Intents," Doctor of Philosophy in Computer Science, University of Arkansas, Fayetteville, University of Arkansas, Fayetteville ScholarWorks@UARK, 2012.

[6] S. Lasya and S. Tanuku, "A Study of Library Databases by Translating Those SQL Queries Into Relational Algebra and Generating Query Trees," 2011.

[7] V. Santos and O. Belo, "Modelling ETL Conciliation Tasks Using Relational Algebra Operators," presented at the 2014 UKSim-AMSS 8th European Modelling Symposium, 2014. 